\title{
Contactless Nanoparticle-Based Guiding of Cells by Controllable Magnetic Fields
}

This article was published in the following Dove Press journal:

Nanotechnology, Science and Applications

\author{
Peter Blümler (D) \\ Ralf P Friedrich (D) ${ }^{2}$ \\ Jorge Pereira ${ }^{3}$ \\ Olga Baun' \\ Christoph Alexiou ${ }^{2}$ \\ Volker Mailänder ${ }^{3,4}$ \\ 'Institute of Physics, Johannes \\ Gutenberg-University Mainz, Mainz, \\ 55।28, Germany; ${ }^{2}$ Department of \\ Otorhinolaryngology, Head and Neck \\ Surgery, Section of Experimental \\ Oncology and Nanomedicine (SEON), \\ Else Kröner-Fresenius-Stiftung- \\ Professorship, Universitätsklinikum \\ Erlangen, Erlangen, 91054, Germany; \\ ${ }^{3}$ Department of Physical Chemistry, Max \\ Planck Institute for Polymer Research, \\ Mainz, 55I28, Germany; ${ }^{4}$ Department of \\ Dermatology, University Medical Center \\ of the Johannes Gutenberg-University \\ Mainz, Mainz, 55I3I, Germany
}

Correspondence: Peter Blümler Institute of Physics, Johannes GutenbergUniversity Mainz, Staudingerweg 7, Mainz, 55।28, Germany

Tel +4961313924240

Fax +4961313925179

Email bluemler@uni-mainz.de
Abstract: Controlled and contactless movements of magnetic nanoparticles are crucial for fundamental biotechnological and clinical research (eg, cell manipulation and sorting, hyperthermia, and magnetic drug targeting). However, the key technological question, how to generate suitable magnetic fields on various length scales $(\mu \mathrm{m}-\mathrm{m})$, is still unsolved. Here, we present a system of permanent magnets which allows for steering of iron oxide nanoparticles (SPIONs) on arbitrary trajectories observable by microscopy. The movement of the particles is simply controlled by an almost force-free rotation of cylindrical arrangements of permanent magnets. The same instrument can be used to move suspended cells loaded with SPIONs along with predetermined directions. Surprisingly, it also allows for controlled movements of intracellular compartments inside of individual cells. The exclusive use of permanent magnets simplifies scaled up versions for animals or even humans, which would open the door for remotely controlled in vivo guidance of nanoparticles or micro-robots.

Keywords: permanent magnets, Halbach, super-paramagnetic, steering, targeting

\section{Introduction}

One of the most auspicious promises of nanomedicine is to replace the current systemic treatment of local diseases to significantly reduce the associated side effects, as large amounts of the drug affect healthy cells or remain mainly in organs, such as liver, kidney, lung. ${ }^{1}$ One way to focus treatment is the application of drugs or nanoparticles functionalized with antibodies or capture proteins that specifically bind to diseased cells or tissues. ${ }^{2,3}$ However, even these approaches are not free of side effects, as a large amount is still captured in non-target organs, tissues or by the reticuloendothelial system. ${ }^{4,5}$ A more site-specific application of pharmaceutical drugs or particles is desirable and urgently needed and could be realized by magnetic drug targeting (MDT). In addition to MDT, where particles must be directed to a specific location, there are other applications where cell guidance is required. ${ }^{6}$ As in the migration and adhesion of cells during embryonic development, tissue formation and wound healing, the targeted guidance of cells is a prerequisite for many areas in regenerative medicine and tissue engineering. Magnetic force-based guidance of cells for separation, isolation, concentration, and patterning has become an important biotechnological tool for cell and tissue bioengineering with low adverse effects on cell viability. ${ }^{7}$ For example, the guidance of magnetically labelled cells enables the manipulation of cells in 2D and 3D cell cultures to form various structures, such as multilayer sheets and spheroids and enables the mimicking of tissue-specific in vivo structures. 
Magnetic stem cell delivery is a promising approach for the treatment of neurodegenerative diseases, myocardial Infarction, cartilage, bone or spinal cord injuries. ${ }^{8-11}$ The cell delivery could be realized by invasive magnetic implants or non-invasive external magnetic systems. However, the exact guiding to the lesion site is still a challenge and a clinical implementation has not yet been developed. Nevertheless, the vision remains to move nanoparticles and nanoparticle-loaded cells noninvasively along predetermined pathways in the human body via suitable magnetic fields. In order to engineer such a magnetic device, several principal and technical problems have to be hurdled.

A magnetic force is given by the gradient of the magnetic flux times the magnetic moment of the object. ${ }^{12}$ However, Gauss's law dictates that a single flux gradient cannot exist in a source-free space and therefore it is difficult to have a bijective force direction. To generate a force that overcomes hydrodynamic friction and even blood flow, the flux gradient as well as the magnetic moment of nano-sized objects must be substantial. Therefore, ferro- or superparamagnetic materials (eg, iron oxides particles) are an obvious choice for the nano-sized objects. $^{13,14}$ Tiny paramagnetic objects in a timeindependent magnetic field align their magnetic moment along the local flux direction. Consequently, magnetic moment and magnetic flux have the same direction and the force always points in the direction of the highest flux (also known as Earnshaw's theorem). An everyday observation that magnets attract ferromagnetic objects (eg, paper clips) in the same way by their south or north pole. Hence, steering requires time-dependent magnet fields. ${ }^{15}$ Based on this reasoning, permanent magnets are not typically considered as useful flux sources for this application, although they offer some important advantages over electromagnets, in particular when the devices need to be scaled up. This is because electromagnets require enormous power for field generation and cooling. Furthermore, the time-dependence of a large coil is far from being linear, as the large inductance of big coils causes severe delays in discharging and charging, making rapid changes in control currents and thus magnetic fields difficult. ${ }^{16}$ Nonetheless, it has been demonstrated that permanent magnets can also be arranged in such a way that the direction and strength of both homogeneous and graded magnetic fields can be changed by simple mechanical rotation. ${ }^{17-19}$

\section{Materials and Methods}

\section{Reagents}

Iron (II) chloride tetrahydrate $\left(\mathrm{FeCl}_{2} \cdot 4 \mathrm{H}_{2} \mathrm{O}\right)$, hydroxylammonium chloride, and Eppendorf ultrafiltration tubes with a molecular weight cut-off (MWCO) of $3 \mathrm{kDa}$ were purchased from Merck (Darmstadt, Germany). Iron reference standards $1 \mathrm{~g} / \mathrm{L}$ were bought from Bernd Kraft $\mathrm{GmbH}$ (Duisburg, Germany). Iron (III) chloride hexahydrate $\left(\mathrm{FeCl}_{3} \cdot 6 \mathrm{H}_{2} \mathrm{O}\right)$, dialysis tubes (Spectrapor 6, MWCO $8 \mathrm{kDa}$ ), sterile Rotilabo ${ }^{\circledR}$ syringe filters with cellulose mixed ester membranes were supplied by Roth (Karlsruhe, Germany). Spectra/Por ${ }^{\circledR} 6$ dialysis tubing with an MWCO of $10 \mathrm{kDa}$ and a diameter of $29 \mathrm{~mm}$ was purchased from Serva Electrophoresis (Heidelberg, Germany). DMEM was purchased from Biochrom (Berlin, Germany), L-glutamine from PAA Laboratories (Pasching, Austria). Endothelial Cell Growth Medium (ECGM) with supplements (C-22010, C-39215) was purchased from PromoCell (Heidelberg, Germany). Water used in all experiments was of ultrapure quality.

\section{Synthesis of in situ-Coated Poly(Acrylic Acid-Co-Maleic Acid) SPIONs (SPION ${ }^{\text {PAM }}$ )}

Poly(acrylic acid-co-maleic acid)-coated superparamagnetic iron oxide nanoparticles $\left(\mathrm{SPION}^{\mathrm{PAM}}\right)$ were synthesized using a previously reported protocol. ${ }^{20,21}$ Briefly, iron oxide nanoparticles were synthesized by coprecipitation of iron (II) and iron (III) at a stoichiometric ratio of 1:2 under argon atmosphere at $80{ }^{\circ} \mathrm{C}$. After purification by dialysis, the particles were coated with PAM. The particles were thereby diluted in a $25 \%(\mathrm{w} / \mathrm{v})$ solution of PAM to a total iron concentration of $5 \mathrm{~g} / \mathrm{L}$. The $\mathrm{pH}$ was adjusted to $\mathrm{pH} 6.5$ using sodium hydroxide solution (5.0 $\mathrm{N}$ ), and the suspension was stirred for $24 \mathrm{~h}$. Excess polymer was removed by multiple centrifugations and resuspension in ultrapure water.

\section{Iron Quantification}

The total iron content was determined using an Agilent 4200 microwave plasma-atomic emission spectrometer (MP-AES, Agilent Technologies, Santa Clara, CA). Three different aliquots $(50 \mu \mathrm{L})$ of the respective ferrofluid were dissolved in $50 \mu \mathrm{L}$ nitric acid $65 \%$, dissolved for 10 min at $95{ }^{\circ} \mathrm{C}$ and diluted with $450 \mu \mathrm{L} \mathrm{H}_{2} \mathrm{O}$. The iron content was then determined with MP-AES using a commercial iron solution as an external standard. The 
calibration curve was prepared with iron concentrations ranging from $0.05 \mathrm{mg} / \mathrm{L}$ to $2.5 \mathrm{mg} / \mathrm{L}$. If the coefficient of determination (R2) in this given range exceeded 0.999 we proceeded with the quantification of the samples.

\section{Magnetic Susceptibility Measurements}

As measure of the magnetizability, the magnetic susceptibility of the particles was determined using a commercial susceptometer (MS2G; Bartington ${ }^{\circledR}$ Instruments, Oxon, UK) and normalized to the iron content of the suspension. The magnetic susceptibility of Resovist ${ }^{\circledR}\left(7.72 \cdot 10^{-3}\right)$ and Rienso $^{\circledR}\left(1.49 \cdot 10^{-3}\right)$ was analyzed as a reference.

\section{Dynamic Light Scattering (DLS) and Zeta Potential Measurements}

The hydrodynamic sizes and Zeta potentials in water and cell culture media were determined using a Malvern Zetasizer Nano ZS (Malvern Instruments, Worcestershire, England). The particles were diluted to a total iron concentration of $30 \mu \mathrm{g} / \mathrm{mL}$ with ultrapure water and measured in triplicates at $25^{\circ} \mathrm{C}$.

\section{Commercially Available Magnetic Nanoparticles}

Magnetic nanoparticles were purchased from Micromod (Germany) labelled with a red fluorescent dye (Ex: 552 $\mathrm{nm} / \mathrm{Em}: 580 \mathrm{~nm}$ ). The particles are $100 \mathrm{~nm}$ in diameter, non-functionalized (plain surface) of dextran-iron oxide composite particles. The particles were kept at $4{ }^{\circ} \mathrm{C}$ and are stable in aqueous buffer solutions $\mathrm{pH}>4$ as the cell culture medium or PBS.

\section{Cells and Culture Conditions}

Normal human dermal fibroblasts (NHDFs) and primary human umbilical vein endothelial cells (HUVECs) were purchased from PromoCell (Heidelberg, Germany). NHDFs were cultured in DMEM with $2 \mathrm{mM}$ L-glutamine and 10\% FCS. HUVECs were used at passage 2-4 and cultivated in ECGM with supplements at 37 ${ }^{\circ} \mathrm{C}$ and $5.0 \% \mathrm{CO}_{2}$. For further passaging, trypsinisation was performed according to the manufacturer's instructions. The murine macrophage cell line RAW 264.7 was bought from ATCC (Wesel, Germany) expanded as a master cell bank and maintained in RPMI-1640 medium supplemented with $10 \%$ fetal bovine serum (FBS) and 100 $\mathrm{U} \mathrm{mL}^{-1}$ penicillin (all from Gibco, Germany) and stored at $37{ }^{\circ} \mathrm{C}$ with $5 \% \mathrm{CO}_{2}$ in an incubator.

\section{Preparation of Cell-Based Experiments}

Cells were seeded into $25 \mathrm{~cm}^{2}$ cell culture flasks in a total volume of $5 \mathrm{~mL}$. The amount of seeded cells was calculated to achieve a final confluency of $70 \%$ after $24 \mathrm{~h}$. After $24 \mathrm{~h}, \mathrm{SPION}^{\mathrm{PAM}}$ were added to a final concentration of $0.5,1,2$ and $4 \mu \mathrm{g}_{\mathrm{Fe}} / \mathrm{mL}$ cell culture media which corresponds to $0.1,0.2,0.4$ and $0.8 \mu \mathrm{g}_{\mathrm{Fe}} / \mathrm{cm}^{2}$ cell culture plate area. The negative control contained $0 \mu \mathrm{g}_{\mathrm{Fe}} / \mathrm{mL}$ cell culture media. For particle uptake, cells were incubated for another $24 \mathrm{~h}$. Subsequently, the samples were washed twice in PBS, harvested, resuspended in $3 \mathrm{~mL}$ cell culture media, and transferred into $4 \mathrm{~cm}$ tissue culture dishes (maximum size for magnets M2 and M3, see Supplementary Table S1). Before the magnetic experiments, cell suspensions were used to determine the absolute cell counts and viability with the $\mathrm{MUSE}^{\circledR}$ Cell Analyzer (Merck-Millipore, Billerica, USA), as well as for SPION quantification measurements using AES spectroscopy (see below). All cell-based experiments were performed in three independent experiments.

For the cell uptake experiments in Raw264.7 cells were seeded at a density of 100000 cells/well in 24-well-plates (Greiner Bio-One, Germany) or at a density of 25000 cells/slide in an iBidi $\mu$-Slides (iBidi, Germany) one day before the incubation with magnetic nanoparticles to attach to the surface of the plate. For the analysis of suspension cells, these were seeded at the same day of the incubation.

Raw264.7 cells incubated with $500 \mu \mathrm{g} / \mathrm{mL}$ of magnetic labelled-nanoparticles for 24 hours in RPMI Medium containing $10 \% \mathrm{FBS}$ at $37{ }^{\circ} \mathrm{C}$ with $5 \% \mathrm{CO}_{2}$ in an incubator. After the incubation, adherent cells were washed with PBS and detached from the culture vessel with $0.25 \%$ trypsin (Gibco, Germany), centrifuged at $300 \mathrm{~g}$ for 7 minutes and re-suspended in fresh PBS for the analysis. For the adherent cells in the iBidi $\mu$-Slides, those were washed twice with fresh PBS and maintained in PBS for later analysis.

\section{Halbach-Magnet-Based Directed Movement of SPION-Treated Cells}

Before placing the magnet setup M2 into the universal mounting frame K-X of a Zeiss Axio Observer.Z1 (Carl Zeiss AG, Oberkochen, Germany), the used objective was equipped with a $30 \mathrm{~mm}$ adapter to increase the parfocal length to $45 \mathrm{~mm}$ and the light collector under the condenser was removed to give place to magnet M2. Subsequently, the tissue culture dishes containing the cell 
suspension were placed into the magnet. Directly before starting the live documentation, the cell suspension was resuspended by pipetting in order to separate already magnetically formed aggregates. The direction of the magnetic force was periodically changed every minute by $90^{\circ}$ and the resulting movement of the cells was imaged with 14 frames per second. Analysis of cellular direction and velocity was performed using the video analysis and modelling tool Tracker 5.0.6 from Open Source Physics (https://physlets.org/tracker/).

Intracellular localization of the fluorescent, magnetic nanoparticles was imaged using Leica TCS SP5 II on a DMI8 microscope body, which was equipped with an HCX PL APO CS 63x/1.4-0.6 oil-immersion objective. Image processing was done via the Leica LAS AF Software. Alternatively, an Olympus CKX41 microscope equipped with Olympus SC30 camera was used.

\section{Quantification of Cellular SPIONs by MP-AES}

The absolute cellular iron content was determined by MPAES. Two millilitres of the cell suspension used for the Halbach magnet experiments were centrifuged for $5 \mathrm{~min}$ at $1000 \mathrm{~g}$. The supernatants were aspirated and the pellets were stored at $-20{ }^{\circ} \mathrm{C}$ until measurement. The cell pellets were then dissolved in $50 \mu \mathrm{L}$ nitric acid $65 \%$, incubated at $95{ }^{\circ} \mathrm{C}$ for 10 min with a shaking incubator and diluted to $450 \mu \mathrm{L}$ with ultrapure water. The total iron content of the cells was then determined using the MP-AES method which was described above. The iron content was normalized to the total number of cells analysed with the MUSE ${ }^{\circledR}$ Cell Analyzer.

\section{Results and Discussion}

The basic idea in our study is to use permanent magnets for precise displacements of SPIONs by combination of a strong, homogeneous (dipolar) magnetic field $\left(B_{0}\right)$ and a second constantly graded (quadrupolar) field $(\mathrm{d} B / \mathrm{d} r=G)$ superimposed on the first (see Figure 1A-C). The homogeneous field magnetizes and orients all SPIONs evenly, aligns their magnetic moments and thus, transforms themselves into tiny magnets. However, the homogeneous magnetic field does not exert a force on them (see Figure 1E). This force is introduced by the second (quadrupolar) gradient field, which all by itself generates a radial gradient without any directional control other than towards its rim (see Figure 1F). The main point here is that in the combination of both fields the motion of the particles is driven predominantly by the component of the gradient field, which is parallel to the direction of the homogeneous field. As a result, particles are guided with constant force and in a single direction over the entire inner volume of the magnet system (see Figure 1G). This solves the guiding problem! The physical details of this design are explained elsewhere. ${ }^{22}$ The most simple realization of this idea is illustrated in Figure $1 \mathrm{~A}-\mathrm{C}$ by a coaxial arrangement of two Halbach cylinders. ${ }^{23}$ The direction of the force is simply adjusted by varying the angle between quadrupole and dipole (Figure 1C) and additionally the strength of the force can be scaled by adding another quadrupole. If properly designed this will vary the force between zero and twice that of a single quadrupole via mutual rotation. ${ }^{18}$ The experiments presented here were performed in three of such magnet setups (M1 to M3, Supplementary Figure S1; for details see Supplementary Table S1) with different size and properties because they had to fit into different microscopes. The position of the rotatable quadrupoles was changed manually in all experiments presented in this work.

Recently, we have shown, that SPIONs with hydrodynamic diameters as small as $75 \mathrm{~nm}$ can be moved at arbitrary angles with such a magnet setup. ${ }^{22}$ In this work, we extend this principle on the intracellular manipulation of SPIONs as well as to the movement of entire human cells and cell clusters loaded with SPIONs. Furthermore, we present a novel magnet system with two compensating quadrupoles, which allows for removing the magnetic force enabling stop-and-go movements.

First, the capability of the magnet system in terms of steering is demonstrated. Equipping the magnet system in Figure $1 \mathrm{C}$ with a second quadrupole allows not only to move SPIONs in certain directions but also to accelerate them by scaling the magnetic force. Because the quadrupoles are designed such that they both produce essentially the same magnetic flux in the inner volume, an angle of $90^{\circ}$ between them extremely reduces the flux/force of the combined gradient to a negligible level. When the quadrupoles are rotated in this compensating configuration to a different angle relative to the dipole and then the angle between is reduced to $0^{\circ}$ (i e, full gradient/force) the particles can be moved even at sharp angles. This gives complete control to manoeuvre magnetic material in the 2D plane of magnet M1 as demonstrated in Supplementary Video S1 by writing the letters $J G U$ with a clearly visible $1 \mathrm{~mm}$ steel ball. This magnet was used to induce similar movements including stops of SPION-loaded murine Raw264.7 cells 

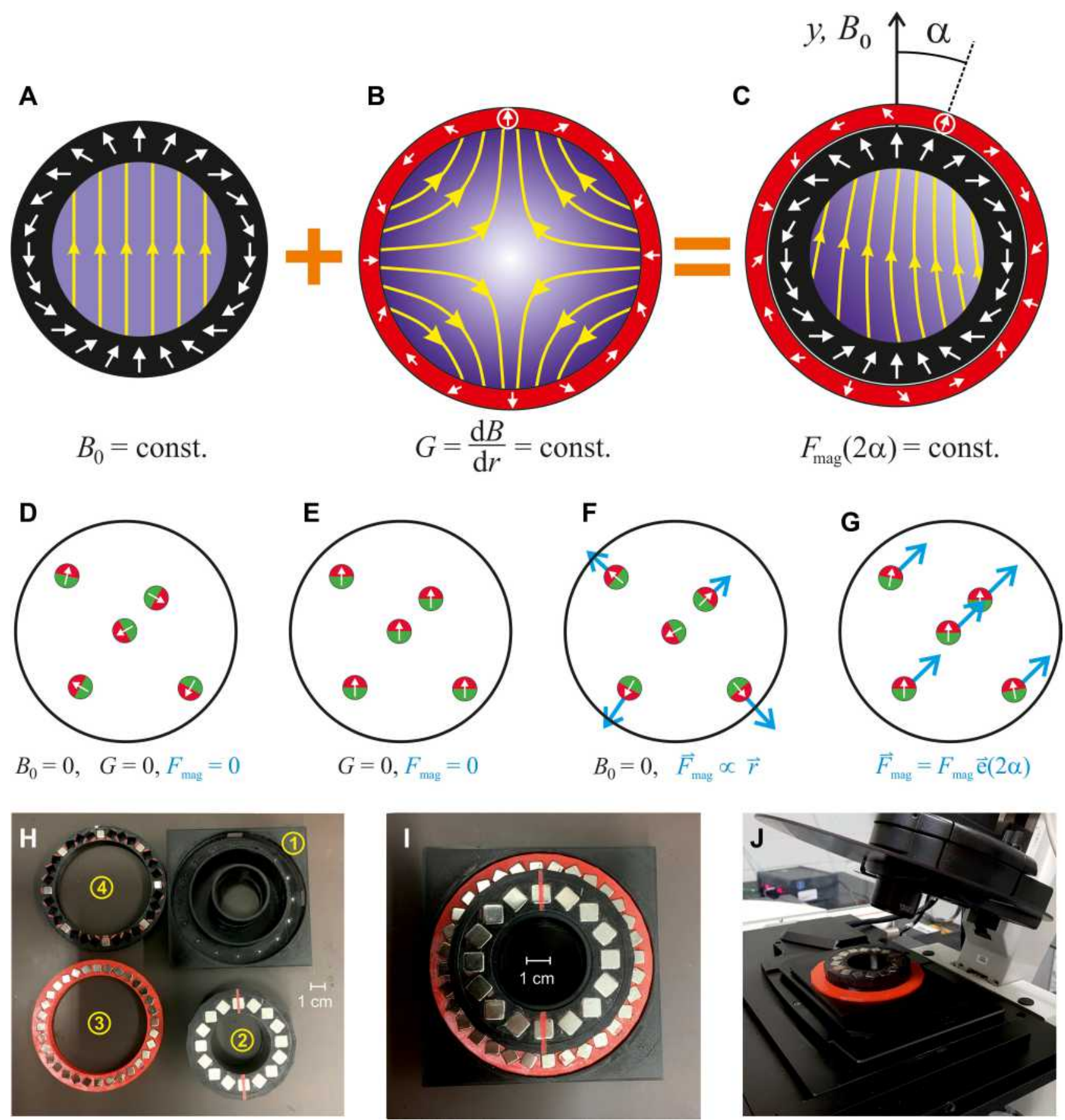

Figure I Schematic cross-sections and architecture of a Halbach magnet compatible for microscope systems. (A) An ideal Halbach dipole cylinder (black) providing a homogeneous magnetic field, $B_{0}$ (magnetic field amplitude is sketched by shades of blue and yellow field lines); (B) An ideal Halbach quadrupole (red) generating a constant radial magnetic field gradient. (C) In a combined Halbach dipole and quadrupole, only the gradient component along $B_{0}$ is relevant to generate the magnetic force $\left(F_{\text {mag }}\right)$, on particles inside the innermost cylinder ( $F_{\text {mag }}$ is homogeneous in strength and direction in a distance $r$ from the center if $B_{0} \gg G r$ is fulfilled). The angle of $F_{\text {mag }}$ changes by $2 \alpha$ if the quadrupole is rotated by $\alpha$ relative to the dipole. For details see. ${ }^{22}$ (D-G) Effect on some magnetic particles in the inner volume. Here it is assumed that the particles have some remnant magnetization, hence they always possess a magnetic moment. (D) In the absence of any magnetic field the particles point in arbitrary directions. (E) In a homogeneous field they align with the field, but in the absence of a gradient there is no force on them. (F) In the gradient field of a quadrupole, the force (blue arrows) is radial, ie, zero in the centre and increasing with the distance from it. (G) the combination of homogeneous and gradient field results in a constant force in one direction. $(\mathbf{H}-$ J) Magnetic guiding system for microscopes. (H) Disassembled system: I) Support to fit microscope stages using different adaptors. 2) Halbach dipole generating $B_{0}=0.097$ T. Red line indicates poles. 3) Halbach quadrupole completely filled with magnets, producing $G=1.3 \mathrm{~T} / \mathrm{m}$. 4) Weaker quadrupole with only every forth magnet present, producing $G=0.33 \mathrm{~T} / \mathrm{m}$. (I) Assembled system with a weight of $273 \mathrm{~g}$. (J) System mounted on a Leica TCS SP5 confocal microscope. 
(Supplementary Figure S2, Supplementary Video S2). The cells were placed inside the magnet with both quadrupoles in compensating position (Supplementary Figure S2A). In absence of a magnetic force, the cells remained at their initial site without clustering. As soon as the cells were moved by switching on the gradients, longer clusters typically in a needle-shaped arrangement appeared (Supplementary Figure S2B-D) which were caused by the mutual dipolar attraction of the SPIONs in adjacent cells. Since the SPIONs are magnetized in the direction of the dipole, the long axis of such clusters is always parallel to the direction of the homogeneous (dipolar) field, $B_{0}$.

Bright field microscopy of SPION-loaded Raw264.7 cells under the influence of magnet M3 demonstrate the formation of magnetically induced needle-formed aggregates of cells containing intra- and extracellular SPIONs (Figure 2). The tendency to form such aggregates increased with increasing SPION-loading per cell. We did not quantify this effect in detail, but it is easily observable within the Supplementary Videos S3 $, \underline{\mathrm{S} 4}, \underline{\mathrm{S} 5}, \underline{\mathrm{S}} 6, \underline{\mathrm{S} 7}$, and $\underline{S 8}$. Such behaviour might be of great relevance for applications where cells should be linearly aligned to form barriers or scaffolds (eg, to direct the growth of capillary or nerve cells in certain directions ${ }^{24}$ ). By comparing Figure 2A (cells before the magnetic fields were applied) with Figure 2D (cells after the application), it can be shown that this aggregation behaviour is reversible.

The magnetic force, $F[\mathrm{~N}]$, on a single SPION (with magnetic moment $m\left[\mathrm{Am}^{2}\right]$ ) in such magnet systems with a magnetic flux gradient, $G[\mathrm{~T} / \mathrm{m}]$, is given by an equation ( $F=m G=M V G)$ demonstrated by Shevkoplyas et al, ${ }^{12}$ where the magnetic moment is expressed by the more common magnetization, $M[\mathrm{~A} / \mathrm{m}]$, and the particle's volume $V^{22}$ However, in a cluster of $n$ SPIONs the force increases up to $n$-fold. ${ }^{25}$ Therefore, the force on a single cell scales more or less with the number of incorporated SPIONs, but in a cell cluster it also depends on the form (eg, a ring or loop vs needle). ${ }^{26}$ Furthermore, the tendency of cluster formation must depend on the exerted mutual dipolar forces between SPIONs and the cells hosting them. Hence, SPION coating and membrane thickness will play a role. Finally, the observed velocity also depends not only on the magnetic force but also on the hydrodynamic properties of the cluster, adhesive friction, and collision with other clusters. This is the reason why we are hesitant to report forces here, because some of these details are difficult to quantify or not known yet. It is also difficult to estimate the forces from the speed of the cell clusters because an analytical hydrodynamic model for this geometry is not available.

In order to analyse the movements of SPION-loaded human cells more systematically, human umbilical vein endothelial cells (HUVECs) and normal human dermal fibroblasts (NHDFs) were incubated for $24 \mathrm{~h}$ with different concentrations of SPIONs. Iron quantification by atomic emission spectroscopy (AES) revealed a linear increase of cell-associated SPIONs with increasing amounts of SPIONs in the cell culture media during incubation (Figure 3). After SPION incubation and subsequent detachment by trypsination, the SPION-loaded cells were placed inside the magnet system M2 and moved on a square path by changing the direction of the quadrupole by $45^{\circ}$ every minute (Figure 4 , Supplementary Figures S3-S7 and Supplementary Videos $\underline{\mathrm{S} 3}, \underline{\mathrm{S} 4}, \underline{\mathrm{S} 5}, \underline{\mathrm{S} 6}, \underline{\mathrm{S} 7}$, and $\underline{\mathrm{S} 8}$ ). The resulting movement at twice that angle (see Figure 1C) of the cells was imaged with 14 frames/s. A detailed analysis of the cellular direction and velocity was performed using the video analysis and modelling tool Tracker 5.0.6 (Open Source Physics). While the magnetically induced average velocity of NHDF cells and
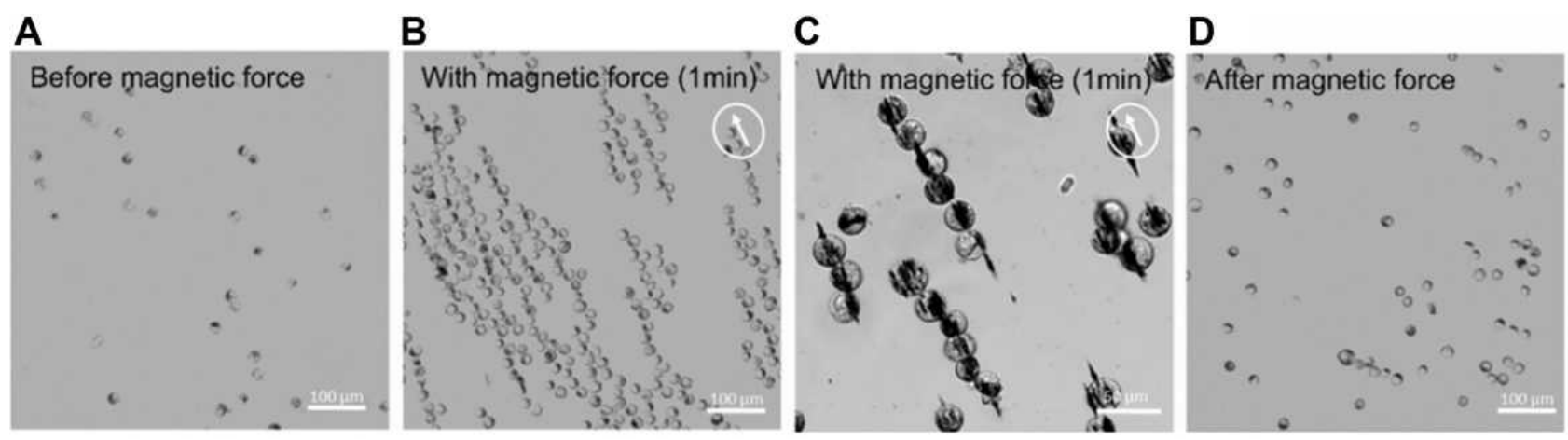

Figure 2 Magnetically based directed cell cluster formation. (A-D) Raw264.7 cells were incubated with magnetic nanoparticles at $500 \mu g / m L$ and analysed after $24 \mathrm{~h}$ incubation in an inverted microscope for magnetically based cluster formation. (B and C) In presence of the magnet system M3, SPION-loaded cells aggregate and form long pole-ladder like structures. (D) These structures were largely reversible, although some of them remained present after removing the magnet system. 

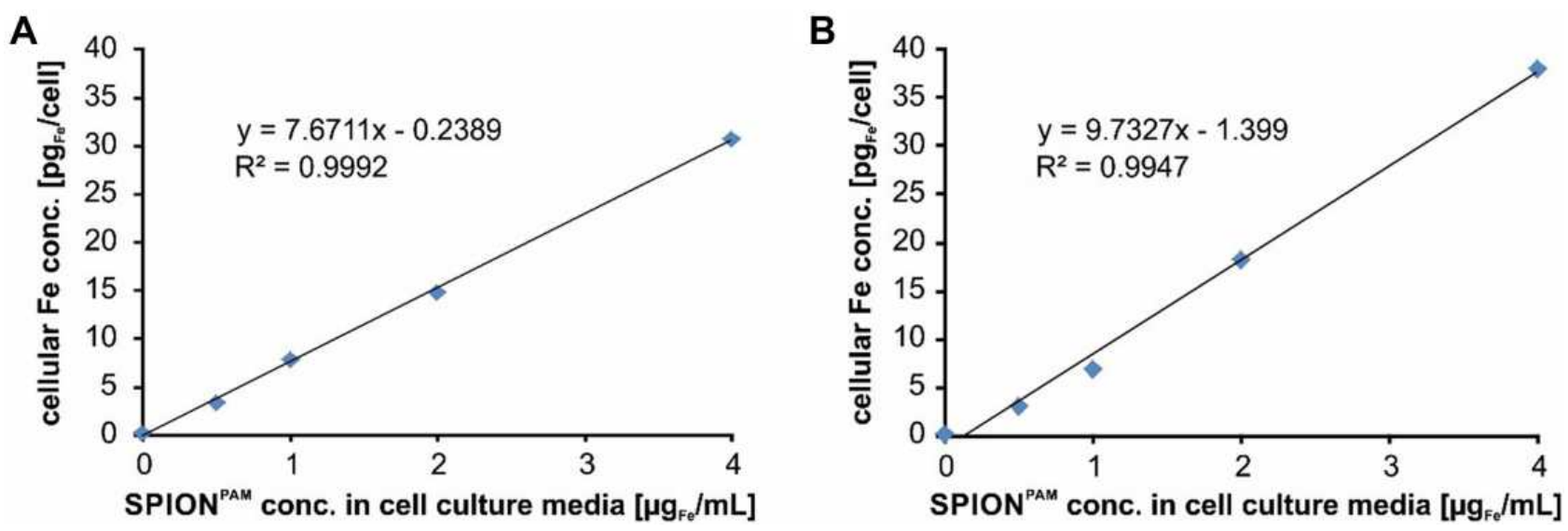

Figure 3 Cellular SPION uptake. To investigate the cellular SPION uptake, HUVECs and NHDF cells were seeded into $25 \mathrm{~cm}^{2}$ cell culture flasks. After $24 \mathrm{~h}$, the cells were incubated for another $24 \mathrm{~h}$ with $0,0.5, \mathrm{I}, 2$, and $4 \mu \mathrm{g}_{\mathrm{Fe}} / \mathrm{mL} \mathrm{SPION}{ }^{\mathrm{PAM}}$. To determine the absolute iron concentration of cells, we analysed the cell suspensions to determine the absolute cell counts with the MUSE ${ }^{\circledR}$ Cell Analyzer and subsequently quantified the iron amount using AES spectroscopy. (A) In HUVECs the iron amount increased from 3.2, 7.7, 14.8, and $30.6 \mathrm{pg}_{\mathrm{Fe}} /$ cell and (B) in NHDF cells, the iron amount increased from 3.0, 6.8, 18.2, and $37.9 \mathrm{pg} \mathrm{Fe}_{\mathrm{Fe}} /$ cell revealing a similar amount and linear uptake or cell association of SPIONs in the two cell lines. R2 represents the coefficient of determination. $y$ describes the mathematic relationship between SPION concentration within the cell culture media and cellular iron content.

A

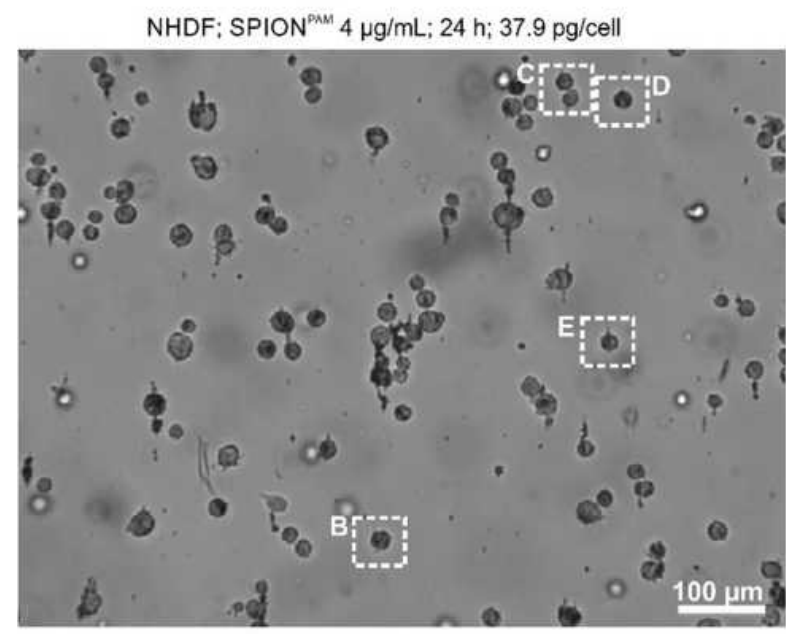

B

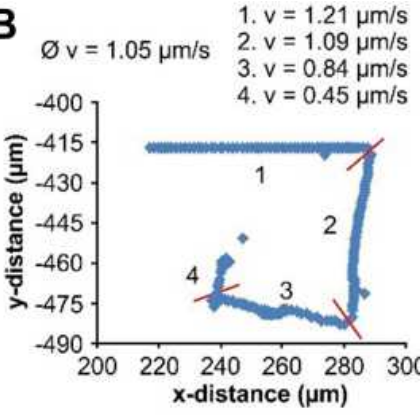

D

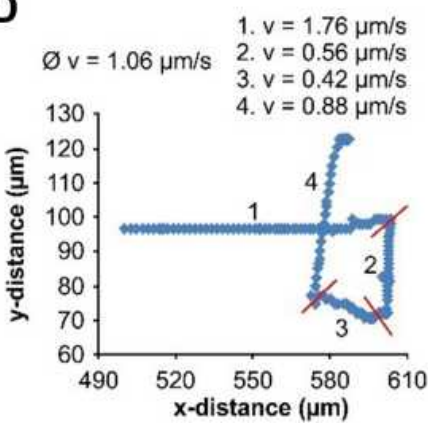

C 1. $v=0.78 \mu \mathrm{m} / \mathrm{s}$ $\varnothing v=1.06 \mu \mathrm{m} / \mathrm{s} \quad \begin{aligned} & 2 . v=1.12 \mu \mathrm{m} / \mathrm{s} \\ & 3, v=0.79 \mu \mathrm{m} / \mathrm{s}\end{aligned}$

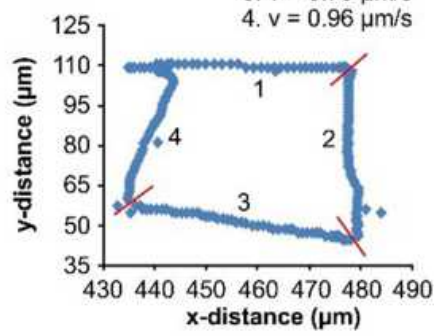

E
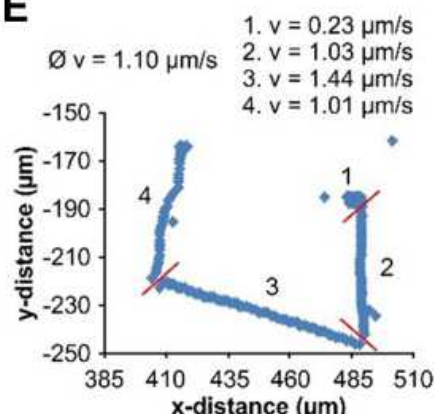

Figure 4 Contact-free movement of SPION-loaded cells. (A) Starting frame of the Supplementary Video S5 used for cell tracking of NHDF cells incubated for 24 h with 4 $\mu \mathrm{g} / \mathrm{mL}$ SPION ${ }^{\text {PAM }}$. The length of the videos is 4 minutes and after each minute the orientation of the quadrupole magnet was turned by $45^{\circ}$, which changes the direction of the magnetic force by $90^{\circ}$. (B-E) Cell tracking of the 4 different cells, marked in Figure 3A by dashed rectangles. $\varnothing \mathrm{v}$, average velocity; during the first, second, third, and fourth minute (I.v, 2.v, 3.v, 4.v); red bars, changing point of the magnetic force direction. Quantification was performed using the video analysis and modelling tool Tracker 5.0.6 from Open Source Physics.

cell clusters increased only slightly with increasing SPION loading, HUVECs incubated with $4 \mu \mathrm{g} / \mathrm{mL}$ SPIONs exhibited significantly increased velocity compared with NHDFs (Figure 5). Because cellular SPION loading after incubation with $4 \mu \mathrm{g} / \mathrm{mL}$ SPIONs showed similar values in HUVECs and NHDFs (30.6 pg/cell and $37.9 \mathrm{pg} /$ cell, respectively) (Figure 3), the average velocity depends not only on the
SPION concentration but also on the cell type. Despite a linear increase of cell-associated SPIONs, the velocity of SPION-containing cells did not increase accordingly, but showed a trend. Most likely, several factors, such as individual cellular SPION-load, adhesive friction, remaining or newly expressed surface proteins for cell attachment and collision with attaching or slow moving cells or cell clusters, 


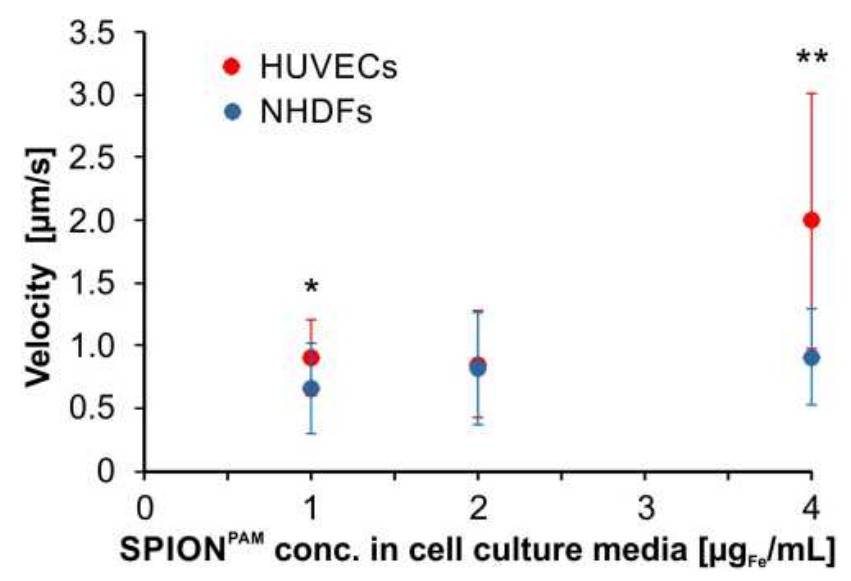

Figure 5 Summary of the magnetically induced velocities of SPION-loaded HUVEC and NHDF cell and cell clusters. HUVECs and NHDFs were incubated for $24 \mathrm{~h}$ with I $\mu \mathrm{g} / \mathrm{mL}$ to $4 \mu \mathrm{g} / \mathrm{mL}$ SPION ${ }^{\text {PAM }}$. After SPION incubation, SPION-loaded cells were detached from the surface by trypsinization, placed inside the magnet system $M 2$ and moved on a square path by changing the direction of the quadrupole by $45^{\circ}$ every minute. Velocities were determined from Supplementary Videos S3, S4, S5, $\mathrm{S6}, \mathrm{S7}$, and S8. Data are expressed as standard deviation of the velocity of $\overline{16}$ cell and cell clusters. Statistical significances between SPION-loaded HUVEC and NHDF cells are indicated with * and **. The respective confidential intervals are $p \leq 0.05$ and $p \leq 0.0005$, respectively, and were calculated via Student's $t$-test.

rearrangement of the SPIONs inside the cells after changing the field direction, together with inhomogeneities of the magnetic field complicate the situation and demand a more detailed study.

So far, the magnetic effects of cell-associated SPIONs were only studied by magnetically based movements of nonattached cells or clusters. To investigate the movement of intracellular SPION, we used RAW264.7 cells, a mouse macrophage-like cell line, loaded with fluorescencelabelled SPIONs. After adherence of the cells to the surface, the magnetic force of magnet M3 was not capable to induce a cellular movement. However, the SPIONs were clearly oriented in the direction of the magnetic force (see Supplementary Figure S8). Moreover, to investigate whether the SPIONs could be directed by solely changing the orientation of the homogeneous field $B_{0}$, the quadrupole of the magnet M3 was removed, so that a strong magnetic force was no longer present. Without the quadrupole, the SPIONs still formed chains, which were aligned by the homogeneous field of the dipole (Figure 6). Interestingly, the SPIONs inside the cells strictly followed the rotation of the dipole, so that they were moved through the cytoplasm. This stirring effect can of course be combined with the steering option of the system. By this, intracellular compartments, such as SPIONs-loaded endosomes, can be moved inside a cell. Potentially this gives the opportunity to give the intracellular compartments a directional distribution. We foresee that this could be interesting for producing or inducing polarized cell types. Polarized cell types are typically lining two compartments like the gastrointestinal tract. Here one side is being exposed to the enzymatically digested food (luminal side) and the other facing the blood (basal or basolateral side). Therefore, transporters of food ingredients need to be differentially present on the luminal and basolateral side.

\section{Conclusion}

In Supplementary Video S1, we demonstrated complete 2D control by writing the initials of the University of Mainz with a small (still visible) steel ball in a very viscous silicone oil. Moreover, this magnetic system offers complete steering control for SPIONs or SPION-loaded cells or cell clusters. The great advantage of using only permanent magnets for the device is the complete lack of electrical power for the generation and control of the magnetic fields. Of course a computer controlled version of this system will use servos to move the magnetic rings, but the power for this will still be small compared to the power needed to generate magnetic fields of similar strength. ${ }^{27}$ Furthermore, it is much easier to scale
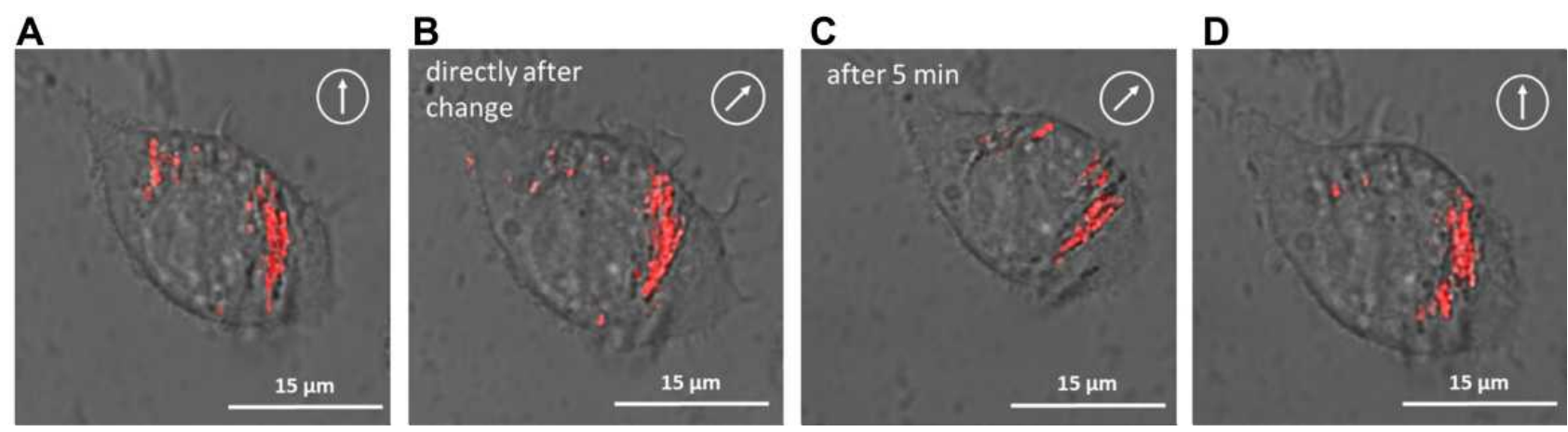

Figure 6 Magnetically based intracellular SPION reorganization. (A-D) Raw264.7 cells were incubated with fluorescent magnetic nanoparticles at $500 \mu$ g/mL and analysed after $24 \mathrm{~h}$ incubation by confocal laser scanning microscopy (cLSM) under a magnetic field at $0^{\circ}, 45^{\circ}$ and $0^{\circ}$, respectively (white encircled arrow). For this experiment, the quadrupole of magnet system M3 was removed and only the dipole was rotated. 
permanent magnet systems, because using larger magnets essentially means adding more material while larger electromagnets have to cope with larger inductance, power, and heat management. The use of permanent magnets for controlled movement of magnetic particles was countered mainly by the argument that the magnetic attraction is always towards the poles and that the magnetic field cannot be switched off. We have proved the opposite with the instrumentation introduced in. ${ }^{22}$ We are not the first who have managed to steer magnetic particles with permanent magnets (see review ${ }^{28}$ ) but our magnet system has the great advantage that the magnetic force is constant over the inner volume which simplifies use and scaling its size (an estimation for a whole body system is given $\mathrm{in}^{22}$ ). There are no moving robotic arms or moving magnets with substantial forces between them. The rotation of the magnetic rings in our device is almost force free. The use of a homogeneous alignment field also causes clustering of the particles which strongly increases the force. Principally the system is not limited to 2D but 3D operation was not demonstrated yet.

The fact that 2D-steering not only works with SPIONs but also with cells, which are associated to or have incorporated SPIONs, opens up new applications. Cells can be guided to specific locations in the body to introduce a directed and structured growth of tissue or to affect diseased tissues or tumors. So far, we have only demonstrated the magnetic systems using in vitro experiments. However, in vivo applications are potentially possible but might be hampered by higher viscosities, strong blood flow (eg, in larger vessels), and complicated paths and boundaries. Especially, the visualization of the guiding procedure is highly challenging. However, magnetically based imaging of SPIONs is possible since those particles, especially super paramagnetic iron oxide nanoparticles used in our experiments, have been extensively investigated as contrast agents for magnetic resonance imaging (MRI). ${ }^{29,30}$ Similarities between MRI, which need a strong homogeneous field and variable magnetic field gradient indicate the principal possibility that such magnet setups cannot only steer SPIONs but also image them. ${ }^{18,31,32}$ Additionally, such magnet systems can also be used to move other super para- or ferromagnetic objects used in micro-fluidics, nanoand micro-robotics ${ }^{33}$ or nanoscience. ${ }^{34}$

\section{Acknowledgments}

R.F. and C.A. want to thank the Else Kröner-FreseniusStiftung (2018_A88), Bad Homburg v.d.H., Germany, and the Dr. Georg and $\mathrm{Lu}$ Zimmermann Foundation, Thannhausen, Germany, for their support. P.B and O.
B. want to acknowledge support and funding by DFGSonderforschungsbereich SFB 1066 and by the University of Mainz (Inneruniversitäre Forschungsförderung). We are very thankful to Prof. U. Häfeli (University of British Columbia, Vancouver) and Prof. R. Zentel (University of Mainz) for checking and improving this manuscript.

\section{Disclosure}

Dr Peter Blümler reports a patent O. Baun, P. Blümler, "Vorrichtung zur Bewegung von magnetischen Partikeln in einem Raum mittels magnetischer Kräfte” DE102016014192A1, 2016 licensed to Sekels GmbH. Prof. Dr. Volker Mailänder reports payment for granted right for patent application by Sekels Company, outside the submitted work; in addition, Prof. Dr. Volker Mailänder has a patent "Contactless movement of cells" with royalities paid. The authors report no other potential conflicts of interest in this work.

\section{References}

1. Tietze R, Lyer S, Dürr S, et al. Efficient drug-delivery using magnetic nanoparticles - biodistribution and therapeutic effects in tumour bearing rabbits. Nanomed. 2013;9(7):961-971. doi:10.1016/j. nano.2013.05.001

2. Mahmoudi M, Laurent S, eds. Iron Oxide Nanoparticles for Biomedical Applications: Synthesis, Functionalization and Application. Elsevier Ltd.; 2018.

3. Thanh NTK. Clinical Applications of Magnetic Nanoparticles: From Fabrication to Clinical Applications. Boca Raton: CRC Press; 2018.

4. Donaghy H. Effects of antibody, drug and linker on the preclinical and clinical toxicities of antibody-drug conjugates. MAbs. 2016;8 (4):659-671. doi:10.1080/19420862.2016.1156829

5. Alphandéry E. Biodistribution and targeting properties of iron oxide nanoparticles for treatments of cancer and iron anemia disease. Nanotoxicology. 2019;13(5):573-596. doi:10.1080/17435390.2019.1572809

6. Ross CL. The use of electric, magnetic, and electromagnetic field for directed cell migration and adhesion in regenerative medicine. Biotechnol Prog. 2017;33(1):5-16. doi:10.1002/btpr.2371

7. Yaman S, Anil-Inevi M, Ozcivici E, Tekin HC. Magnetic force-based microfluidic techniques for cellular and tissue bioengineering. Front Bioeng Biotechnol. 2018;6:192. doi:10.3389/fbioe.2018.00192

8. Kubinová Š. Biomaterials and magnetic stem cell delivery in the treatment of spinal cord injury. Neurochem Res. 2020;45 (1):171-179. doi:10.1007/s11064-019-02808-2

9. Kodama A, Kamei N, Kamei G, et al. In vivo bioluminescence imaging of transplanted bone marrow mesenchymal stromal cells using a magnetic delivery system in a rat fracture model. $J$ Bone Joint Surg Br. 2012;94(7):998-1006. doi:10.1302/0301-620X.94B7.28521

10. Kamei G, Kobayashi T, Ohkawa S, et al. Articular cartilage repair with magnetic mesenchymal stem cells. Am J Sports Med. 2013;41 (6):1255-1264. doi:10.1177/0363546513483270

11. Kamei N, Ochi M, Adachi N, et al. The safety and efficacy of magnetic targeting using autologous mesenchymal stem cells for cartilage repair. Knee Surg Sports Traumatol Arthrosc. 2018;26 (12):3626-3635. doi:10.1007/s00167-018-4898-2 
12. Shevkoplyas SS, Siegel AC, Westervelt RM, et al. The force acting on a superparamagnetic bead due to an applied magnetic field. $L a b$ Chip. 2007;7(10):1294-1302. doi:10.1039/b705045c

13. Tombácz E, Turcu R, Socoliuc V, et al. Magnetic iron oxide nanoparticles: recent trends in design and synthesis of magnetoresponsive nanosystems. Biochem Biophys Res Commun. 2015;468:442-453. doi:10.1016/j.bbrc.2015.08.030

14. Alphandéry E. Applications of magnetotactic bacteria and magnetosome for cancer treatment: a review emphasizing on practical and mechanistic aspects. Drug Discov Today. 2020;25(8):1444-1452. doi:10.1016/j.drudis.2020.06.010

15. Nacev A, Komaee A, Sarwar A, et al. Towards control of magnetic fluids in patients: directing Therapeutic Nanoparticles to Disease Locations. IEEE Control Syst Mag. 2012;32(3):32-74. doi:10.1109/ MCS.2012.2189052

16. Probst R, Lin J, Komaee A, et al. Planar steering of a single ferrofluid drop by optimal minimum power dynamic feedback control of four electromagnets at a distance. J Magn Magn Mater. 2011;323 (7):885-896. doi:10.1016/j.jmmm.2010.08.024

17. Bauer C, Raich HP, Jeschke G, et al. Design of a permanent magnet with a mechanical sweep suitable for variable-temperature continuous-wave and pulsed EPR spectroscopy. J Magn Reson. 2009;198(2):222-227. doi:10.1016/j.jmr.2009.02.010

18. Blümler P. Proposal for a permanent magnet system with a constant gradient mechanically adjustable in direction and strength. Concepts Magn Reson Part B Magn Reson Eng. 2016;46(1):41-48. doi: $10.1002 / \mathrm{cmr} . b .21320$

19. Leupold HA, Potenziani E, Tilak AS. Adjustable multi-tesla permanent magnet field sources. IEEE Trans Magn. 1993;29(6):2902-2904. doi: $10.1109 / 20.281092$

20. Friedrich RP, Zaloga J, Schreiber E, et al. Tissue plasminogen activator binding to superparamagnetic iron oxide nanoparticle-covalent versus adsorptive approach. Nanoscale Res Lett. 2016;11(1):297. doi:10.1186/s11671-016-1521-7

21. Tóth IY, Illés E, Bauer RA, et al. Designed polyelectrolyte shell on magnetite nanocore for dilution-resistant biocompatible magnetic fluids. Langmuir. 2012;28(48):16638-16646. doi:10.1021/la302660p

22. Baun O, Blümler P. Permanent magnet system to guide superparamagnetic particles. J Magn Magn Mater. 2017;439:294-304. doi:10.1016/j.jmmm.2017.05.001
23. Halbach K. Design of permanent multipole magnets with oriented rare earth cobalt material. Nucl Instrum Methods. 1980;169(1):1-10. doi:10.1016/0029-554X(80)90094-4

24. Falconieri A, De Vincentiis S, Raffa V. Recent advances in the use of magnetic nanoparticles to promote neuroregeneration. Nanomed. 2019;14(9):1073-1076. doi:10.2217/nnm-2019-0103

25. Schaller V, Wahnström G, Sanz-Velasco A, et al. Effective magnetic moment of magnetic multicore nanoparticles. Phys Rev B. 2009;80 (9):092406. doi:10.1103/PhysRevB.80.092406

26. Singamaneni S, Bliznyuk VN, Binek C, et al. Magnetic nanoparticles: recent advances in synthesis, self-assembly and applications. J Mater Chem. 2011;21(42):16819-16845. doi:10.1039/c1jm11845e

27. Tretiak O, Blümler P, Bougas L. Variable single-axis magnetic-field generator using permanent magnets. AIP Adv. 2019;9(11):115312. doi:10.1063/1.5130896

28. Yang Z, Zhang L. Magnetic actuation systems for miniature robots: a review. Adv Intell Syst. 2020;2000082:1-18.

29. Sharifi S, Seyednjad H, Laurent S, et al. Superparamagnetic iron oxide nanoparticles for in vivo molecular and cellular imaging. Contrast Media Mol Imaging. 2015;10:329-335.

30. Unterweger H, Dezsi L, Matuszak J, et al. Dextran-coated superparamagnetic iron oxide nanoparticles for magnetic resonance imaging: evaluation of size-dependent imaging properties, storage stability and safety. Int $J$ Nanomed. 2018;13:1899-1915. doi:10.2147/IJN.S156528

31. Zimmerman Cooley C, Stockmann JP, Armstrong BD, et al. Twodimensional imaging in a lightweight portable MRI scanner without gradient coils. Magn Reson Med. 2015;73(2):872-883. doi:10.1002/ mrm. 25147

32. O'Reilly T, Teeuwisse WM, de Gans D, Koolstra K, Webb AG. In vivo 3D brain and extremity MRI at $50 \mathrm{mT}$ using a permanent magnet Halbach array. Magn Reson Med. 2021;85(1):495-505. doi: $10.1002 / \mathrm{mrm} .28396$

33. Diller E, Sitti M. Micro-Scale mobile robotics. Found Trends Robot. 2011;2:143-259. doi:10.1561/2300000023

34. Ditter D, Blümler P, Klöckner B, et al. Microfluidic synthesis of liquid crystalline elastomer particle transport systems which can be remote-controlled magnetically. Adv Funct Mater. 2019;1902454:1-12.
Nanotechnology, Science and Applications

\section{Publish your work in this journal}

Nanotechnology, Science and Applications is an international, peerreviewed, open access journal that focuses on the science of nanotechnology in a wide range of industrial and academic applications. It is characterized by the rapid reporting across all sectors, including engineering, optics, bio-medicine, cosmetics, textiles, resource sustainability and science. Applied research into nano-materials, particles, nano-structures and fabrication, diagnostics and analytics, drug delivery and toxicology constitute the primary direction of the journal. The manuscript management system is completely online and includes a very quick and fair peer-review system, which is all easy to use. Visit http://www.dovepress.com/testimonials.php to read real quotes from published authors. 